\title{
Atorvastatin For Reduction Of 28-Day Mortality In Hospitalized COVID-19 Patients: A Protocol For A Randomized, Double-Blinded, Placebo-Controlled, Clinical Trial
}

Moataz Maher Emara ( $\nabla$ mm.emara@mans.edu.eg)

Mansoura University Faculty of Medicine https://orcid.org/0000-0001-8911-1812

Neamat Hamdy Elsawy

Ministry of Health and Population

Kholoud M. Abdelaaty

Mansoura university

Amal salah Elhamaky

Ministry of Health and Population

Naglaa Hamdi Eltahan

Ministry of Health and Population

\section{Research Article}

Keywords: COVID-19, SARS-CoV-2, coronavirus, statins, hmg coa, atorvastatin, mortality

Posted Date: February 10th, 2022

DOI: https://doi.org/10.21203/rs.3.rs-1268956/v1

License: (c) (1) This work is licensed under a Creative Commons Attribution 4.0 International License.

Read Full License 


\section{Title}

Atorvastatin for reduction of 28-day Mortality in Hospitalized COVID-19 Patients: A Protocol for a Randomized, Double-blinded, Placebo-Controlled, Clinical Trial

\section{Names protocol contributors}

Moataz Maher Emara'1 MD, EDAIC, ORCID: 0000-0001-8911-1812

Neamat Hamdy Elsawy², Pharm D

Kholoud M. Abdelaaty ${ }^{3}$, BCPS

Amal Salah Elhamaky ${ }^{4}$

Naglaa Hamdi Eltahan ${ }^{5}$

\section{Affiliations:}

1. Mansoura University Faculty of Medicine, Department of Anesthesiology and Intensive Care and Pain Medicine

2. Fowa Central Hospital, Ministry of Health and Population, Egypt

3. Specialized Medical Hospital, Mansoura university, Egypt

4. Mansoura Specialized Hospital, Ministry of Health and Population, Egypt

5. Sherbin central hospital, Ministry of Health and Population, Egypt

\section{Corresponding author:}

Moataz M. Emara, MD, EDAIC

Mansoura University, Faculty of Medicine

Department of Anesthesiology and Intensive Care and Pain Medicine

60 Elgomhoria st, Mansoura, Egypt. Postal code. 35516

Email: mm.emara@mans.edu.eg, Tel. +201064048848 


\section{Abstract}

- Background: Although mass vaccination has reduced the severity of COVID-19, mortality is still high among hospitalized patients. Being a sepsis-like disease, an antiinflammatory drug as atorvastatin would reduce mortality and severity in COVID-19.

- Methods: We designed a randomized clinical trial that recruited 220 COVID-19 patients admitted in the COVID-19 isolation hospital at Mansoura University, Egypt. 110 cases were assigned to receive $40 \mathrm{mg}$ Atorvastatin once daily for 28 days and 110 were assigned to receive placebo. Delta Pharm company supported the study with the drug and the placebo, which mimics the drug as regards the drug package, the tablet color, consistency, and size. All patients received the standard treatment as per the hospital protocol.

- The Institutional Review Board approval and the informed consent from all participants were obtained.

The primary outcome is the 28-day all-cause mortality. Additionally, we will collect the in-hospital mortality, the need for mechanical ventilation, time to clinical improvement, in-hospital thrombo-embolic events, acute kidney injury, and the hospital and the intensive care duration of stay. We plan to follow the patients up for 6 months for reporting mortality and long-term neurological, psychological, and respiratory consequences.

We will report the un-adjusted 28-mortality using $X^{2}$. Then, we will report the adjusted odds ratio with a pre-planned multiple logistic regression model. We will report our results using the point estimate and the 95\% confidence interval and the P-value.

- Discussion: After summarizing the main results of the study, we will discuss the outcomes with the current evidence. In addition to the planned regression model results, we would report any potential effect modifications after the planned subgroup analysis by the disease severity and the invasive mechanical ventilation status. In the end, we will conclude the risk reduction of atorvastatin on the 28-day all-cause mortality and the main secondary outcomes.

- Trial registration: The study was registered at the Clinical Trial Registry (NCT04952350) on July $1^{\text {st }}$, 2021. https://clinicaltrials.gov/ct2/show/NCT04952350 


\section{Keywords}

COVID-19; SARS-CoV-2; coronavirus; statins, hmg coa; atorvastatin; mortality 


\section{Administrative information}

Trials guidance: please include this text in your protocol just above the Administrative information table:

Note: the numbers in curly brackets in this protocol refer to SPIRIT checklist item numbers. The order of the items has been modified to group similar items (see http://www.equator-network.org/reportingguidelines/spirit-2013-statement-defining-standard-protocol-items-for-clinical-trials/).

\begin{tabular}{|c|c|}
\hline Title $\{1\}$ & $\begin{array}{l}\text { Atorvastatin for reduction of 28-day Mortality in } \\
\text { Hospitalized COVID-19 Patients: A Randomized, Double- } \\
\text { blinded, Placebo-Controlled, Clinical Trial }\end{array}$ \\
\hline Trial registration $\{2 \mathrm{a}$ and $2 \mathrm{~b}\}$. & $\begin{array}{l}\text { NCT04952350 at ClinicalTrials.gov } \\
\text { https://clinicaltrials.gov/ct2/show/NCT04952350 }\end{array}$ \\
\hline Protocol version $\{3\}$ & V03. 1st Jul 2021 \\
\hline Funding $\{4\}$ & $\begin{array}{l}\text { The company that will provide the research group with the } \\
\text { drug and the placebo will not support additional funds or } \\
\text { interests. The investigators declare no relevant conflict of } \\
\text { interest. }\end{array}$ \\
\hline Author details $\{5 \mathrm{a}\}$ & $\begin{array}{l}\text { 1. Moataz Maher Emara, MD, EDAIC } \\
\text { Mansoura University, Faculty of Medicine, Egypt } \\
\text { 2. Neamat Hamdy Elsawy, Pharm D } \\
\text { Fowa Central Hospital, Ministry of Health and } \\
\text { Population, Egypt } \\
\text { 3. Kholoud M. Abdelaaty, BCPS } \\
\text { Specialized Medical Hospital, Mansoura university, } \\
\text { Egypt } \\
\text { 4. Amal Salah Elhamaky }\end{array}$ \\
\hline
\end{tabular}




\begin{tabular}{|l|l|}
\hline \multirow{5}{*}{$\begin{array}{l}\text { Mansoura Specialized Hospital, Ministry of Health } \\
\text { and Population, Egypt } \\
\text { 5. Naglaa Hamdi Eltahan } \\
\text { Sherbin central hospital, Ministry of Health and } \\
\text { Population, Egypt }\end{array}$} \\
\hline $\begin{array}{l}\text { Name and contact information for } \\
\text { the trial sponsor \{5b\} }\end{array}$ & $\begin{array}{l}\text { Mansoura University } \\
\text { Address: Elgomhouria St., Mansoura City, Egypt } \\
35516 \\
\text { Email: mua@ @mans.edu.eg } \\
\text { Tel. }+20 \text { (50) 2383781 / +20 (50) 2397055 / +20 (50) } \\
2397054, \text { FAX: }+20 \text { (50) 239733 / +20 (50) 2397900 } \\
\text { Website: http://www.mans.edu.eg }\end{array}$ \\
\hline Role of sponsor \{5c\} & $\begin{array}{l}\text { The sponsor only supports the research conducted } \\
\text { through institutional resources. }\end{array}$ \\
\hline
\end{tabular}

\section{Introduction}

\section{Background and rationale $\{6 \mathrm{a}\}$}

COVID-19 has been a major global health problem. Despite mass vaccination, waves of epidemics are still striking. SARS-CoV-2 has infected more than 265 million cases all over the world. By December 5th, 2021, COVID-19 caused more than 5.2 million deaths worldwide, and approximately 20,919 deaths in Egypt. ${ }^{1}$ COVID-19 increases the risk of pneumonia, cardiovascular, and thromboembolic events. ${ }^{2}$

COVID-19 implies an aggressive immune reaction - the cytokine storm. ${ }^{3}$ Elevated cytokines - as interleukin-6 (IL-6) - activate the nuclear factor kappa B (NF-KB) pathway, which causes 
sepsis, capillary damage, acute pulmonary injury, severe acute respiratory distress (ARDS), multi-organ damage, and death. ${ }^{3,4}$ COVID-19 survivors suffered the post-COVID syndrome, which includes pulmonary, cardiovascular, renal, and neuropsychiatric consequences. These long-term effects may result from a direct viral infection, systemic inflammation, neuroinflammation, microvascular thrombosis, and neurodegeneration. ${ }^{5}$

$\beta$-Hydroxy $\beta$-methyl glutaryl-CoA (HMG-CoA) drugs - Statins - are lipid-lowering drugs with pleiotropic effects. Statins might benefit COVID-19 patients due to their anti-inflammatory and antioxidant effects: (1) bind to the SARS-CoV-2 main protease and prevent invasion of the host cells; ${ }^{6}(2)$ activate autophagy and regulate SARS-CoV-2 virus degradation or replication, which may reduce viral load; (3) modulate the immune response by blocking the NF-KB pathway and NLRP3 inflammasomes, so theoretically decrease the cytokine storm in COVID-19; ${ }^{6}$ (4) improve the endothelial function, which participates in the pathogenesis of the COVID-19. ${ }^{7}$

Statins propose antithrombotic and anticoagulant effects. In a chemical-induced venous thrombosis in a murine model, atorvastatin enhanced the vein thrombus resolution. ${ }^{8}$ In addition, using statins was associated with a lower risk of recurrent pulmonary embolism (PE) by $50 \%{ }^{7}$

In influenza pneumonia, studies suggest the survival benefits of statins. One study found that statins were associated with a 0.59 reduction in the odds of death before or during hospitalization. ${ }^{9}$ Another study found a hazard ratio of death of 0.41 with a matched sample. ${ }^{10}$ Besides, simvastatin significantly improved the 28-day survival in hyperinflammatory ARDS. ${ }^{11}$ COVID speaking, statins reduced mortality by almost half with an adjusted hazard ratio (HR) of 0.58 or slowed the progression of death. ${ }^{12,13}$

As regards the potential neuroprotective effects of statins, they reduced the risk of Alzheimer's disease and related dementia with HR; 0.54 (0.32-0.91), and 2.45 (0.69-8.68) compared to non-statin users. ${ }^{14}$ Statins also have neuroprotective benefits by regulating cholesterol metabolism in the brain. ${ }^{15}$

Consequently, we designed a randomized clinical trial to investigate the role of atorvastatin in hospitalized (severe and critical cases) COVID-19 patients on the 28-day mortality and 
the 6-month long-term consequences. We hypothesized that atorvastatin $40 \mathrm{mg}$ once daily for 28 days would reduce the 28-day all-cause mortality in adult hospitalized COVID-19 patients.

\section{Objectives $\{7\}$}

\section{The study Hypothesis:}

Atorvastatin $40 \mathrm{mg}$ per day for 28 days would reduce the 28-day all-cause mortality in adult hospitalized (severe and critical) COVID-19 patients.

\section{The Objective of the study:}

To test the efficacy of atorvastatin $40 \mathrm{mg}$ per day in reducing 28-day mortality and the 6month consequences in hospitalized patients with severe and critical COVID19.

\section{Trial design $\{8\}$}

We wrote the protocol according to the recommendations of the Standard PRotocol Items for clinical Trials (SPIRIT 2013) guidelines. ${ }^{16}$

Study design: Double-blind randomized (1:1) placebo-controlled trial

\section{Methods: Participants, interventions and outcomes}

\section{Study setting $\{9\}$}

Mansoura University COVID-19 Isolation Hospital

\section{Eligibility criteria $\{10\}$}

\section{Inclusion criteria:}

We included adult patients ( $\geq 18$ years old) with severe or critical COVID-19 admitted to the COVID-19 Mansoura University isolation hospital. We included patients who are PCRconfirmed, clinically or radiologically diagnosed with COVID-19.

Cases are defined as severe or critical according to the WHO definition ${ }^{17}$; where severe cases have the clinical signs of severe pneumonia and $\mathrm{SpO} 2<90 \%$ on room air, or RR $>30$ breaths/min without any critical criteria, while critical cases have ARDS, or sepsis, septic shock or pulmonary embolism, acute coronary syndrome, or acute stroke. 


\section{Exclusion criteria:}

Exclusion criteria were chronic statin use, serum creatine kinase $(C K)>5$ times the upper limit of normal (ULN), serum transaminases $>5$ times ULN, acute hepatic failure, chronic liver disease (Child-Pugh Classification C), history of rhabdomyolysis or myopathies, severe renal impairment not receiving renal replacement therapy (estimated $\mathrm{Cr} \mathrm{cl}<30 \mathrm{ml} / \mathrm{min}$ ), pregnant and lactating women, patients who are expected to die within 48 hours, or patients on chronic colchicine, cyclosporine, or ritonavir.

\section{Who will take informed consent? \{26a\}}

We secured written informed consent from the patient or the legal representative if the patient was unable to give consent. The IRB of Mansoura University - Faculty of Medicine approved the protocol. (Supplement S1)

One of the investigators conducted the consent process with the patient or the legal representative by signing two copies (one for the investigator and one for the patient). The consent was signed and dated by the conducting investigator and the consent provider.

Patients or their legal representatives were informed clearly that all patients have the same chances to receive the active treatment or the placebo.

We provided the informed consent form as a supplement to this protocol. (Supplement S2)

\section{Additional consent provisions for collection and use of participant data and biological specimens $\{26 \mathrm{~b}\}$}

The patient consent discussed the patients' publication of their anonymous data. The biological specimens were handled according to the institution's protocol. (Supplement S2)

\section{Interventions}

\section{Explanation for the choice of comparators $\{6 b\}$}

Placebo: Patients in the placebo group received the standard treatment protocol according to the local guidelines. The placebo resembles the original drug regards the drug package, the tablet color, consistency, and size.

Atorvastatin has not proved its efficacy yet, so there is no harm in not receiving atorvastatin in COVID19 patients, according to the Declaration of Helsinki 2008, paragraph $32 .{ }^{18}$ 


\section{Intervention description $\{11 \mathrm{a}\}$}

Patients were randomized to receive either placebo or enteral atorvastatin $40 \mathrm{mg} / \mathrm{day}$ for 28 days.

Administration in unconscious or ventilated patients: The patients received the drug through a nasogastric tube.

\section{Criteria for discontinuing or modifying allocated interventions $\{11 b\}$}

The safety assessment included daily monitoring for adverse events; we checked CK level if the patient developed myalgia or unexplained weakness and stopped if $\mathrm{CK}>10$ times ULN, drug-induced hepatitis, or serum transaminases $>8$ times ULN. Patients who received azole antifungals had a lower threshold for monitoring of liver injury and rhabdomyolysis.

\section{Strategies to improve adherence to interventions $\{11 \mathrm{c}\}$}

Patients started the drug during hospitalization, and, on discharge, they received the estimated amount of their study drug and were educated to complete the 28-day treatment. Additionally, we contacted them after their discharge to ensure adherence to treatment.

\section{Relevant concomitant care permitted or prohibited during the trial $\{11 \mathrm{~d}\}$}

All patients received the standard of care according to local hospital protocol. Antiviral treatment was allowed and reported.

\section{Provisions for post-trial care $\{30\}$}

We will conduct a six-month follow-up via telephone and at the outpatient clinic. An interview with all participants will be face-to-face by trained physicians and asked to complete a series of questionnaires, including a self-reported symptom questionnaire. We will report a detailed 6-months follow-up data later.

\section{Outcomes $\{12\}$}

Primary outcome: all-cause mortality will be recorded within 28 days after randomization. We will ascertain mortality by a telephone call or the national death index. 


\section{Secondary outcomes:}

\section{A) Early outcomes:}

1. Need for invasive mechanical ventilation,

2. Invasive mechanical ventilation and oxygen support duration (days)

3. Time to clinical improvement (defined as 2 points reduction in the WHO disease ordinal progression scale ${ }^{19}$ or discharge, whatever happens first)

4. Serious adverse effect leading to drug discontinuation

5. ICU and hospitalization length of stay (days)

6. CRP on days $3,7,14$, and 28

7. SOFA score on days $3,7,14$, and 28

8. COVID19 WHO disease progression scale on days $3,7,14$, and 28

9. Incidence of acute kidney injury (AKI), defined as an increase in Scr by $\geq 0.3$ $\mathrm{mg} / \mathrm{dl}$ in 48 hour or Increase in Scr by $\geq 50 \%$ in 7 days or Oliguria for $\geq 6$ hour. 20

10. In-hospital Deep vein thrombosis or PE.

11. Mortality at hospital discharge

CRP, D-dimers, SOFA scores, WHO disease progression score, and AKI were evaluated only during the hospitalization period.

B) Late outcomes: (will be published in a separate report)

1. 6- month all-cause mortality

2. long-term outcomes of 6 months post-acute COVID19 syndrome

- pulmonary function tests (PFTs), high-resolution computed tomography of the chest, and incidence of cognitive impairment.

- health-related quality of life (anxiety or depression), and re-hospitalization rates.

- extrapulmonary organ function (including eGFR, and ultrasonographic features of kidney, and liver).

\section{Participant timeline $\{13\}$}

We started enrolment on August $17^{\text {th }}, 2021$ and finished recruitment on October $6^{\text {th }}, 2021$. The ascertainment of the primary outcome for the last enrolled patient was on November $2^{\text {nd }}$. Figure 1 shows the timeline of the study. 


\section{Sample size $\{14\}$}

A sample size of 97 patients in each group would achieve at least $80 \%$ power to detect a risk difference of $0.2(20 \%)$ in the 28-day all-cause mortality (primary outcome) between the null hypothesis (both proportions are 0.6 ) and the alternative hypothesis (the proportion in the non-statin group would be 0.4 ). We assumed a significance level ( $\alpha$ ) of 0.05 and used the Chi-square test of independent proportions in MedCalc software.

To compensate for the estimated loss-to-follow-up, we increased the sample size to be 110 patients in each group.

The mortality data was estimated from the average mortality in January, February, and March 2021 at the Mansoura University Isolation Hospital among all hospitalized patients.

\section{Recruitment $\{15\}$}

According to the current admission rate at the Mansoura University - Isolation Hospital, the admission rate is 250 cases per month on average; our needed sample is 220 cases. We have already finished patient recruitment.

\section{Assignment of interventions: allocation}

\section{Sequence generation $\{16 \mathrm{a}\}$}

Sequence generation is mentioned with the concealment mechanism.

\section{Concealment mechanism $\{16 \mathrm{~b}\}$}

A pharmacist, not involved in the study, created a randomization table (1:1) using excel random permuted blocks (4, or 6 in each block) and allocated patients to either group to ensure random-sequence concealment. Randomization was conducted within 24 hours after hospital admission after evaluation of the inclusion criteria. We started patient recruitment only after the registration of the study protocol on ClinicalTrial.gov.

Patients were randomized to receive either placebo or atorvastatin $40 \mathrm{mg} /$ day orally for 28 days. All patients received the standard of care according to local hospital protocol. Antiviral treatment was allowed and reported.

Implementation $\{16 \mathrm{c}\}$ 
Randomization table was available only with the pharmacist who assigned each group for the intervention or the control.

\section{Assignment of interventions: Blinding}

\section{Who will be blinded $\{17 a\}$}

Patients, caregivers, data collectors, and data analysts are blinded for the study group. The Delta Pharma company (Egypt) provided the atorvastatin and a similar placebo. The company did not - and will not - participate in any step of the study, including participant recruitment, data collection, data analysis, or results reporting. The company prepared a similar package of drug and placebo (labelled as A and B).

Even the pharmacist - involved in treatment allocation - will not know what the treatment group is, just $A$ or $B$.

\section{Procedure for unblinding if needed $\{17 \mathrm{~b}\}$}

Data Safety and Monitoring Board (DSMB) will be kept blinded for the assignment groups except if the termination of the study is an issue.

For emergency unmasking, one of the directors of Mansoura COVID-19 research council, who will not participate in the study, will have the true labels and the randomization table in a closed envelope. The envelope will be only opened if DSMB decided to unmask for a safety issue.

\section{Data collection and management}

\section{Plans for assessment and collection of outcomes $\{18 \mathrm{a}\}$}

Our primary outcome, 28-day all-cause mortality, is highly objective. We ascertained mortality by a telephone call, or by reviewing the national death index. Outcome assessors were trained to collect the COVID19 WHO disease progression score.

We added the definitions of the outcomes to the paper CRF to guide the outcome assessor while collecting data. Age and study durations were collected as dates (i.e., date of birth, 
date of admission, and date of discharge) to calculate the continuous variable on data cleaning before analysis.

Categorical outcomes were ascertained as continuous outcomes - as appropriate - and were categorized on the data analysis stage. The CRF - with the data and code dictionary - is available as a supplementary file (S3) to the protocol.

\section{Plans to promote participant retention and complete follow-up $\{18 \mathrm{~b}\}$}

Patients started and almost received their intervention within the hospital. We educated patients and emphasized receiving their study drug after hospital discharge and contacted with telephones for follow-up. On the hospital discharge, patients received their expected doses of the assigned intervention.

\section{Data management $\{19\}$}

We collected the data in a paper case report form (CRF) and upload the data into an electronic CRF after 1-2 months of the patient enrolment. Paper CRF with the proposed coding was preprepared (supplement S3), coding for missing data as 9999. Four investigators double-checked the data (2 investigators per 110 cases).

\section{Confidentiality $\{27\}$}

Patient confidentiality will be kept before, during, and after the study. Anonymized CRF papers were uploaded to electronic CRF. After the end of the study, the paper CRF will be kept in the hospital record room - only accessed by the research team after authorization of the IRB.

Plans for collection, laboratory evaluation and storage of biological specimens for genetic or molecular analysis in this trial/future use $\{33\}$

N/A. Laboratory and biological specimens are manipulated according to the institution's protocol. 


\section{Statistical methods}

\section{Statistical methods for primary and secondary outcomes $\{20 \mathrm{a}\}$}

The primary analysis will be based on the intention-to-treat strategy.

Categorical variables will be presented as proportion and percent. Continuous variables will be presented as mean (standard deviation) for parametric data or as a median $\left(25^{\text {th }}-75^{\text {th }}\right.$ percentile) for non-parametric data.

Chi-square test and t-test will be used to conduct a univariate analysis of demographic features correlated with study groups. We will report the $95 \%$ confidence interval and the Pvalue for our statistical tests.

In each group, we will compare the 28-day all-cause mortality rate (primary outcome) using the chi-square test with reporting the rate ratio and $95 \%$ confidence interval.

Statistical analysis will be achieved with SPSS, version 26. The level of statistical significance will be $p$-value $\leq 0.05$.

\section{Interim analyses $\{21 b\}$}

After recruitment of 25,50 , and $75 \%$ of the planned sample size, we performed interim analyses of the in-hospital mortality.

The Data Safety Monitoring Board (DSMB) and the IRB members were blinded for the study allocation. Results of the interim analysis are presented in figure 2. They were timely reported and revised by the IRB and the DSMB.

\section{Methods for additional analyses (e.g. subgroup analyses) $\{20 \mathrm{~b}\}$}

Subgroup analysis will be conducted for the following subset which will be selected 48 hours after recruitment:

a) patients with and without invasive mechanical ventilation

b) severe cases and critically ill cases 
We will run a multiple logistic regression for the association between receiving atorvastatin and 28-day all-cause mortality, controlling for age, gender, the number of comorbidities (DM, HTN, IHD, AF, COPD), and COVID19 severity (severe or critical).

A multi-linear regression model will be used to assess the reduction in ventilation duration, controlling for the same risk factors in the logistic regression model.

Methods in analysis to handle protocol non-adherence and any statistical methods to handle missing data $\{20 \mathrm{c}\}$

We are planning to perform an intention-to-treat analysis, and we will manage missed data by analysing the available data or by multiple imputations - reporting the method used.

Plans to give access to the full protocol, participant level-data and statistical code $\{31 \mathrm{c}\}$

We seek to publish the full protocol; besides, we have uploaded the protocol on the clinical trial registry. The CRF with the statistical code is provided as a supplement (supplement S3) to the protocol.

\section{Oversight and monitoring}

Composition of the coordinating centre and trial steering committee $\{5 \mathrm{~d}\}$ N/A. This study is not a multicenter trial.

Composition of the data monitoring committee, its role and reporting structure $\{21 \mathrm{a}\}$ We had the COVID-19 scientific committee at Mansoura University as the DSMB. The members of the board have no direct role in the study. They monitor and evaluate the study conduct, interim analyses, and adverse events.

\section{Adverse event reporting and harms $\{22\}$}

We planned to report any in-hospital adverse events by the study investigators or the outof-hospital adverse events by the patients. Patients have telephone contacts with WhatsApp for reporting any adverse events or discontinuation of the study drug. 


\section{Frequency and plans for auditing trial conduct $\{23\}$}

N/A. Due to the short period of the study, we did not plan for study audits.

Plans for communicating important protocol amendments to relevant parties (e.g. trial participants, ethical committees) $\{25\}$

We reported the interim analyses results to the IRB and the DSMB. Additionally, we reported the end of the patients' recruitment to the IRB, DSMB, and updated the study status on the Clinical Trial registry.

\section{Dissemination plans $\{31 \mathrm{a}\}$}

We would report our results in the MedRxive preprint while aiming for publication in peerreview journals. We will report the early study outcomes, then the late 6-months outcomes in a separate later-on publication.

\section{Discussion}

We will report the main results of our study. Then, we will discuss the outcome in light of the current evidence. In addition to the planned regression model results, we would report any potential effect modifications after the planned subgroup analysis by the disease severity and the invasive mechanical ventilation status.

\section{Trial status}

This is the third version of the protocol approved by the IRB at Mansoura University, Faculty of Medicine. We started the study recruitment on August $18^{\text {th }}, 2021$, and the patients' recruitment end on October $6^{\text {th }}$.

\section{Abbreviations}

AF, Atrial fibrillation

AKI, acute kidney injury

ARDS, acute respiratory distress syndrome

CARDS, COVID-19 ARDS.

$\mathrm{CK}$, creatine kinase 
COPD, Chronic obstructive pulmonary disease

COVID-19, Coronavirus disease of 2019.

CRF, Case report form

DM, Diabetes mellitus

DSMB, Data and Safety Monitoring Board

HMG-CoA, $\beta$-Hydroxy $\beta$-methylglutaryl-CoA

HTN, hypertension

ICU, intensive care unit

$I H D$, ischemic heart disease

IL-6, interleukin-6

IRB, institutional research board

NF-kB, nuclear factor kappa B

NLRP3, Nod-like receptor pyrin domain containing 3

PAl-1, plasminogen activator inhibitor-1

$\mathrm{PCR}$, polymerase chain reaction

PE, pulmonary embolism

PFTs, pulmonary function tests

$R R$, respiratory rate

SARS, sever acute respiratory syndrome

SARS-COV-2, severe acute respiratory syndrome - coronavirus 2.

SOFA score, sequential organ failure assessment

\section{Declarations}

\section{Acknowledgements}

We acknowledge the support of the Delta Pharm (Egypt) for their providing of the Atorvastatin (Atorstat) and placebo for the study. The company did not participate in any phase of the study, patients' inclusion, data collection, and will not participate in data analysis, or reporting and writing the manuscript. 


\section{Authors' contributions $\{31 \mathrm{~b}\}$}

Neamat Hamdy Elsawy and Naglaa Hamdi Eltahan conceived the idea and contributed the study design and protocol development; Kholoud M. Abdelaaty and Amal Salah Elhamaky contributed the study design and protocol development; Moataz Maher Emara is the lead investigator and led the proposal and protocol development. All authors revised and accepted the final manuscript

Additional investigators have, substantially, contributed to the study. They would be added as co-author to the final reports of the study if they fulfilled the authorship criteria.

\section{Funding $\{4\}$}

We used the institutional resources for conducting the study. Additionally, Delta Pharm company (Egypt) for their providing of the Atorvastatin (Atorstat) and placebo for the purpose of the study.

The company did not - and will not - participate in any phase of the study, including patients' enrollment, data collection, data analysis, or reporting and writing the manuscript.

\section{Availability of data and materials $\{29\}$}

We will make the anonymous individual data available at reasonable request after publication. Patient confidentiality will be kept.

\section{Ethics approval and consent to participate $\{24\}$}

We obtained the IRB approval (code No. R.21.04.1300) at Mansoura University, Faculty of Medicine on June $2^{\text {nd }}, 2021$. (Supplement S1)

Written informed consent was received either from the patient or the legal representative if the patient is unable to provide consent.

One of the investigators conducted the consent process with the patient or the legal representative by signing two copies (one for the investigator and one for the patient). The consent was signed and dated by both the conducting investigator and the patient side. 
Patients or their legal representatives (in the case of unconscious patients) were informed clearly that all patients have the same chances to receive the active treatment or the placebo.

\section{Consent for publication $\{32\}$}

We received a consent for publication along with the consent for participation. (Item 13 in the consent form, supplement S2)

\section{Competing interests $\{28\}$}

All authors report no financial or other competing interests.

\section{Authors' information (optional)}

Moataz Maher Emara, MD, EDAIC is a clinical lecturer of Anaesthesiology and Intensive Care at Mansoura University, Faculty of Medicine. Emara is an active member of the European Association of Anaesthesiology and Intensive Care (EACTAIC) and a member of the Social Network Task Force under the Education Committee. Additionally, Dr. Emara is an associate member of the European Society of Anaesthesiology and Intensive Care (ESAIC).

Neamat Hamdy Elsawy, Pharm D, is the Surveillance director of infectious diseases, Preventive Sector, Fowa Health District, Kafer Elsheikh, Ministry of Health and Population, Egypt. Dr. Elsawy is the Clinical research coordinator, Fowa Central Hospital, Kafer Elsheikh, Ministry of Health and Population, Egypt.

Kholoud M. Abdelaaty, BCPS clinical pharmacist at Specialized Medical Hospital, Mansoura university, and Mansoura University coordinator in Supreme Council of Universities Medication Information Network.

Amal Salah Elhamaky is a clinical pharmacist and clinical research coordinator at Mansoura Specialized Hospital, Specialized Medical Centers, Ministry of Health and Population, Egypt. Naglaa Hamdi Eltahan is a clinical pharmacist at the intensive care unit and clinical research coordinator, Sherbin central hospital, Dakahliya, Ministry of Health and Population, Egypt. 
All the authors have completed the Clinical Research Scholar Training (CSRT) Program at Harvard Medical School - offered as a grant from the Egyptian Ministry of Health and Population.

\section{References}

1. World Health Organization. COVID-19 Weekly Epidemiological Update, Edition 46, published 2021.

2. Mondal S, Quintili AL, Karamchandani K, Bose S. Thromboembolic disease in COVID-19 patients: A brief narrative review. J Intensive Care. 2020;8:1-10.

3. Ragab D, Salah Eldin H, Taeimah M, Khattab R, Salem R. The COVID-19 Cytokine Storm; What We Know So Far. Front Immunol. 2020;11:1-4.

4. Hojyo S, Uchida M, Tanaka K, Hasebe R, Tanaka Y, Murakami M, et al. How COVID-19 induces cytokine storm with high mortality. Inflamm Regen. 2020;40:1-7.

5. Nalbandian A, Sehgal K, Gupta A, Madhavan MV, McGroder C, Stevens JS, Cook JR, Nordvig AS, Shalev D, Sehrawat TS, Ahluwalia N. Post-acute COVID-19 syndrome. Nature medicine. 2021;27:601-15.

6. Díez RR, Tejera-Muñoz A, Marquez-Exposito L, Mateos SR, Santos-Sanchez L, Marchant V, Tejedor-Santamaria L, RAMOS A, Ortiz A, Egido J, Ruiz-Ortega M. Statins: Could an old friend help the fight against COVID-19?. Br J Pharmacol. 2020;177:4873-86

7. Lee KC, Sewa DW, Phua GC. Potential role of statins in COVID-19. Int J Infect Dis. 2020;96:615-7.

8. Bifulco M, Gazzerro P. Statin therapy in COVID-19 infection: Much more than a single pathway. Eur Hear J - Cardiovasc Pharmacother. 2020;6:410-1.

9. Vandermeer ML, Thomas AR, Kamimoto L, Reingold A, Gershman K, Meek J, et al. Association between use of statins and mortality among patients hospitalized with laboratoryconfirmed influenza virus infections: A multistate study. J Infect Dis. 2012;205:13-9.

10. Laidler MR, Thomas A, Baumbach J, Kirley PD, Meek J, Aragon D, Morin C, Ryan PA, Schaffner W, Zansky SM, Chaves SS. Statin treatment and mortality: propensity scorematched analyses of 2007-2008 and 2009-2010 laboratory-confirmed influenza hospitalizations. In Open forum infectious diseases. 2017;2: 2633851.

11. Calfee CS, Delucchi KL, Sinha P, Matthay MA, Hackett J, Shankar-Hari M, et al. Acute respiratory distress syndrome subphenotypes and differential response to simvastatin: secondary analysis of a randomised controlled trial. Lancet Respir Med. 2018;6:691-8. 
12. Zhang XJ, Qin JJ, Cheng X, Shen L, Zhao YC, Yuan Y, et al. In-Hospital Use of Statins Is Associated with a Reduced Risk of Mortality among Individuals with COVID-19. Cell Metab. 2020;32:176-187.

13. Rodriguez-Nava G, Trelles-Garcia DP, Yanez-Bello MA, Chung CW, Trelles-Garcia VP, Friedman HJ. Atorvastatin associated with decreased hazard for death in COVID-19 patients admitted to an ICU: A retrospective cohort study. Crit Care. 2020;24:4-5.

14. Lee JW, Choi EA, Kim YS, Kim Y, You HS, Han YE, et al. Statin exposure and the risk of dementia in individuals with hypercholesterolaemia. J Intern Med. 2020;288:689-98.

15. Kim SW, Kang HJ, Jhon M, Kim JW, Lee JY, Walker AJ, et al. Statins and inflammation: New therapeutic opportunities in psychiatry. Front Psychiatry. 2019;10:103.

16. Chan A-W, Tetzlaff JM, Altman DG, Laupacis A, Gøtzsche PC, Krleža-Jerić K, Hróbjartsson A, Mann H, Dickersin K, Berlin J, Doré C, Parulekar W, Summerskill W, Groves T, Schulz K, Sox H, Rockhold FW, Rennie D, Moher D. SPIRIT 2013 Statement: Defining standard protocol items for clinical trials. Ann Intern Med 2013;158:200-207.

17. World Health Organization. COVID-19 clinical management: living guidance, 25 January 2021. World Health Organization; 2021.

18. Puri KS, Suresh KR, Gogtay NJ, Thatte UM. Declaration of Helsinki, 2008: implications for stakeholders in research. Journal of postgraduate medicine. 2009;55:131

19. Marshall JC, Murthy S, Diaz J, Adhikari NK, Angus DC, Arabi YM, Baillie K, Bauer M, Berry S, Blackwood B, Bonten M. A minimal common outcome measure set for COVID-19 clinical research. The Lancet Infectious Diseases. 2020; 20:192-7.

20. Kellum JA, Lameire N, Aspelin P, Barsoum RS, Burdmann EA, Goldstein SL, et al. Kidney disease: Improving global outcomes (KDIGO) acute kidney injury work group. KDIGO clinical practice guideline for acute kidney injury. Kidney Int Suppl. 2012;2:1-138. 
Figures

\section{Figure 1}

Schedule of enrolment, interventions, and assessments according to the SPIRIT template for the study timepoints

\section{Figure 2}

Interim analyses of the in-hospital mortality analyzed using $X 2$ at $25 \%, 50 \%$, and $75 \%$ of patients, reporting the percent on the $Y$ axis and the number/total within the bars 